\title{
Stellar Populations in KDCs of Sa Galaxies
}

\author{
Jesús Falcón-Barroso ${ }^{1,2}$, Roland Bacon ${ }^{3}$, Michele Cappellari ${ }^{4}$, Roger \\ Davies $^{4}$, P. Tim de Zeeuw ${ }^{2}$, Eric Emsellem ${ }^{3}$, Davor Krajnović ${ }^{4}$, \\ Harald Kuntschner ${ }^{5}$, Richard M. McDermid ${ }^{2}$, Reynier F. Peletier ${ }^{6}$, \\ Marc Sarzi ${ }^{7}$ and Glenn van de Ven ${ }^{8}$
}

\footnotetext{
${ }^{1}$ European Space and Technology Centre, Keplerlaan 1, 2200 AG Noordwijk, The Netherlands email: jfalcon@rssd.esa.int

${ }^{2}$ Sterrewacht Leiden, University of Leiden, NL-2333 CA Leiden, The Netherlands

${ }^{3}$ Observatoire de Lyon, 9 av. Charles André, F-69230 Saint-Genis Laval, France

${ }^{4}$ Sub-Department of Astrophysics, University of Oxford, Oxford OX1 3RH, UK

${ }^{5}$ ST-ECF, European Southern Observatory, D-85748 Garching bei München, Germany

${ }^{6}$ Kapteyn Astronomical Institute, University of Groningen, NL-9700 AV Groningen,

The Netherlands

${ }^{7}$ Centre for Astrophysics Research, University of Hertfordshire, Hatfield, UK

${ }^{8}$ Institute for Advanced Study, Einstein Drive, Princeton, NJ 08540, USA
}

\begin{abstract}
We present integral-field observations of kinematically decoupled components (KDCs) in a sample of 24 Sa galaxies part of the SAURON Survey. We show how the V/ $\sigma$ maps can be used to highlight the presence of the KDC and investigate their stellar populations (i.e. age) to understand their origin (i.e. secular vs hierarchical scenarios). We find that KDCs can be long-lived and are typically well aligned with the galaxies kinematical major axis, supporting mounting evidence that secular evolution becomes important at intermediate redshifts. Dynamically colder ones seem to host younger stellar populations. We also relate these features to the ones found in our sample of 48 elliptical and lenticular galaxies. We find that there is no age vs size relation for these decoupled components in Sa galaxies, suggesting that the dominant factor determining their size is the amount of star formation they have been subject to during their life time.
\end{abstract}

Keywords. galaxies: bulges, galaxies: spiral, galaxies: stellar content

\section{Introduction}

The search for kinematically decoupled components in galaxies has been one of the major endeavors astronomers have been pursuing in the last decade (e.g. Forbes, Franx \& Illingworth 1994; Carollo et al. 1997). For many years KDCs have been used as tracers of the sequence of merging events that we believe have led to the galaxies we see in the sky today, and therefore they have quickly become one of the most important proofs for the validity of hierarchical formation models (e.g. Lacey \& Cole 1993). Under this picture, spiral galaxies are the result of interactions that, with time, have built up both their bulge and disks components, and occasionally KDCs if the merging event had some significance. Nevertheless, as shown via numerical simulations (Pfenniger \& Norman 1990), the presence of a disk gives these galaxies the opportunity to evolve internally (more than early-type galaxies), and give rise to new structures (e.g. spiral arms, bars). In this secular evolutionary scenario the formation of a KDC is the result of the redistribution of gas towards the inner regions of the galaxies which then settled in a new dynamically distinct component that, in turn, led to the formation of new stars. 

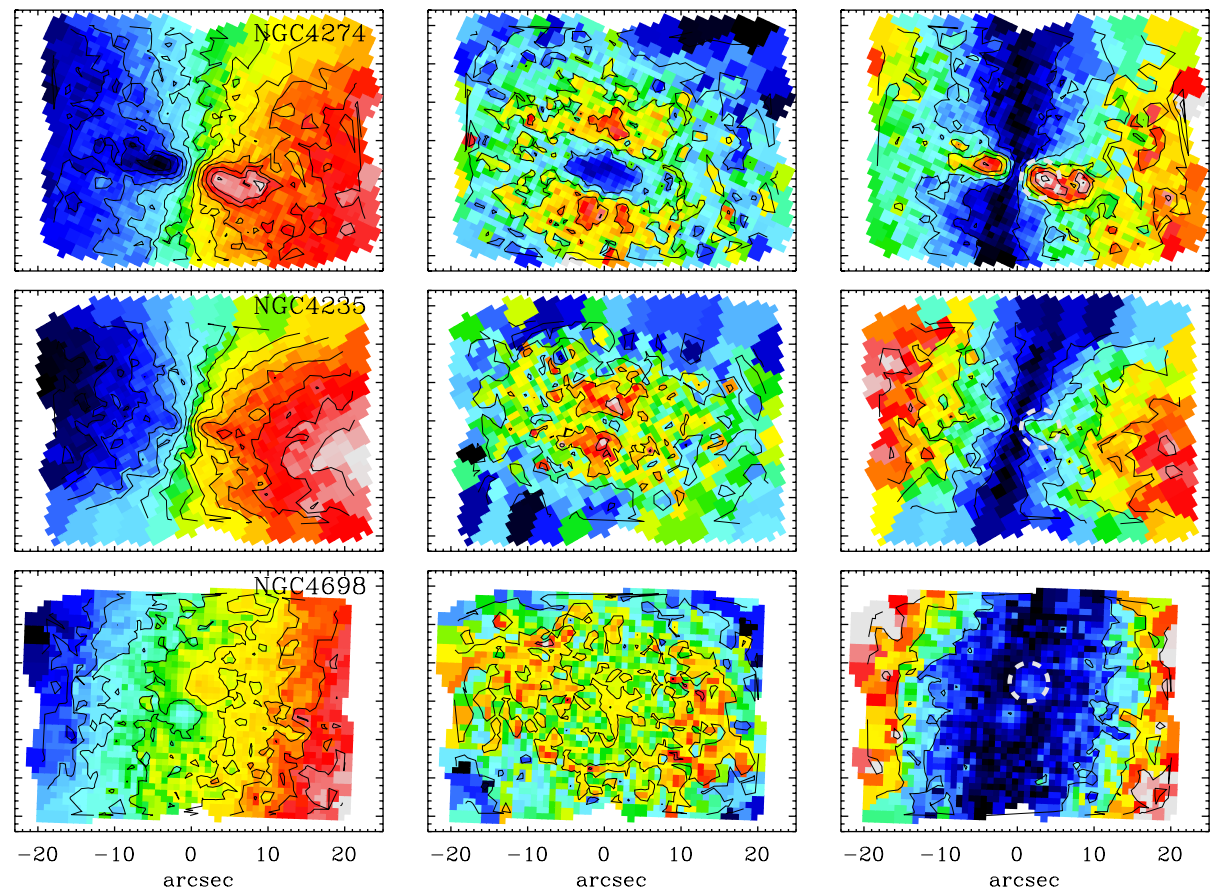

Figure 1. Velocity, velocity dispersion and V/ $\sigma$ maps for three of the 12 galaxies with KDCs in our sample of 24 Sa galaxies. Top row: NGC4274, middle: NGC4235, bottom: NGC4698. White open circles over the $\mathrm{V} / \sigma$ maps indicate the apertures size and locations used to derive the parameters in Figure 2.

Given the two competing scenarios, the main issues are: can we distinguish between the two scenarios for a KDC in a spiral galaxy today? When does secular evolution become significant in spiral galaxies? Part of the answer to these questions have already been addressed by photometric studies of galaxies at intermediate redshifts (e.g. Jogee et al. 2004). Here, we use the spectroscopic information delivered by the SAURON integral-field spectrograph to gain further insight.

\section{Identifying decoupled components in integral-field maps}

Our starting point is the kinematic maps of the sample of 24 Sa galaxies in the SAURON survey presented in Falcón-Barroso et al. (2006). The maps were carefully inspected to detect the presence of any kinematic substructure (i.e. different from the overall rotation of the galaxy as defined by its main disk) embedded in the centres of the galaxies. The identification of the decoupled components is mainly done on the line-of-sight velocity maps. KDCs usually show up as a twist of the zero-velocity curve in the very center, in which case the decoupled component is misaligned with respect to the galaxies main rotation axis, or as a sudden rise of the velocity along the major axis of the galaxies compared with the surrounding velocity field. In recent years it has also become customary (e.g. Emsellem et al. 2001; Márquez et al. 2003) to look for the presence of dynamically cold components based on a drop of velocity dispersion (i.e. sigma-drop) in the inner regions of the galaxies. The appearance of this feature additionally highlights the dynamically hotter nature of the surrounding bulge.

As one may suspect, the key aspect for the detection of such a KDC is the amplitude of its rotation compared to its surroundings. In many cases the difference is small and it 

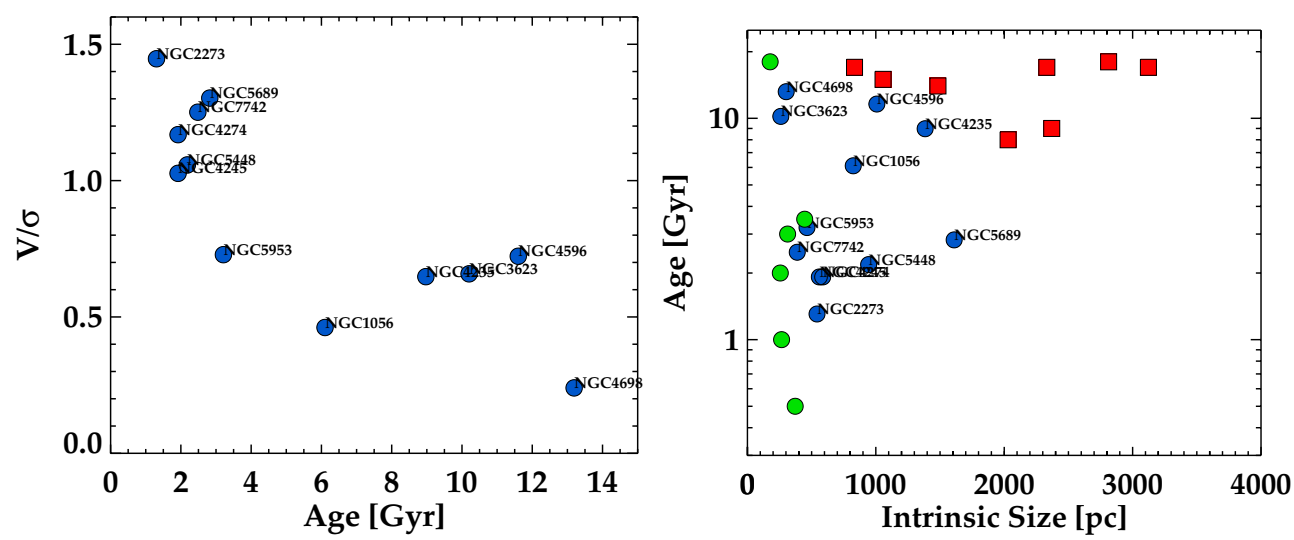

Figure 2. Left: Average $\mathrm{V} / \sigma$ measured, within 1 arcsec radius aperture, at the location of maximum amplitude in the KDC vs the average age measured at the same locations. Right: Average age vs intrinsic size for the KDCs in our sample (blue solid circles), and small and big KDCs in McDermid et al. (2006) (green solid circles, and red squares respectively).

shows up as a slight pinch of the isovelocity contours, which makes it difficult to find. An alternative way to highlight their presence is the use of both the velocity and velocity dispersion information simultaneously. In Figure 1 we show the velocity, velocity dispersion and the local $\mathrm{V} / \sigma$ map (defined as the ratio of the absolute value of the radial velocity over the velocity dispersion) for three galaxies with KDCs in our sample. In the top row we present the case of NGC4274 which displays a fast rotating inner component in the radial velocity field, also quite apparent from the sigma-drop in the velocity dispersion map. In the middle row we show the case of NGC4235, where it is difficult to detect the KDC from the velocity field (i.e. just a pinching of the isovelocity contours), but whose presence is confirmed first by the velocity dispersion, and is greatly enhanced by the $\mathrm{V} / \sigma$ map. Finally in the bottom row, we present the case of NGC4698, one of the two misaligned KDCs in our sample. Here the evidence for the KDC comes from the velocity field alone, as there is no signature in the velocity dispersion map.

\section{Stellar populations of KDCs in Sa galaxies}

In order to determine the KDCs stellar populations, we first measured line-strength index maps for the galaxies in our sample (i.e. $\mathrm{H} \beta$, [OIII] and $\mathrm{Mg} b$ ) on the Lick system (see Peletier et al. (2007), but also these proceedings). Then we derived a stellar age, metallicty and abundance ratio maps in the way described in Mcdermid et al. (2006). Finally, we measured the average age within a 1 radius arcsec aperture centred at the location of the KDC (see dashed open circles is Figure 1). The measured quatities are the luminosity weighted values along the line-of-sight, and therefore are related to the KDCs, but also to the underlaying population. Since the line-of-sight kinematics is dominated by the $\mathrm{KDC}$, it is reasonable to assume that the populations measured here are also representative of the KDC. We have, additionally, determined their intrinsic size as their length measured from the $\mathrm{V} / \sigma$ map. In this contribution we base our discussion on the results coming from the stellar age only.

In Figure 2 (left) we show the relation between the measured $\mathrm{V} / \sigma$ at the KDC versus the average age at the same location. One can see that there is a trend such that dynamically colder KDCs (i.e. higher $\mathrm{V} / \sigma$ ) tend to be younger than those with lower 
$\mathrm{V} / \sigma$ values. The fact that most of the galaxies in this subset displays KDCs that are flatened, well aligned and in co-rotation with the main body of the host galaxy (with the exception of NGC4698 and NGC5953), suggests that these cold components may have an internal origin and that they have been the result of continous inflow of gas to the inner regions, that settles in a disk and from which stars can then form. The above trend extending towards old KDCs may indicate the significance of secular evolution already at intermediate redshifts, although a clean differentiation with respect to hierarchical merging is not yet possible. In addition, it is important to remark that, at this stage, it is difficult to assess which is the dominant factor driving this relation: the fading of the KDC's stellar population, or the dynamical heating of the KDC over time.

In Figure 2 (right) we reproduce the plot already presented in McDermid et al. (2006), for the KDCs in the E and S0 galaxies in the SAURON sample, but this time adding the KDCs found in the Sa galaxies. Contrary to the sample of early types, where there were two classes of KDCs, we find that the KDCs found here lie in between the two groups, without showing any particular trend between age and size (although note that that co-rotating KDCs in the E/S0 sample are not included in this figure). Considering that spiral galaxies contain much more gas, it is likely that the final size and age of the KDCs in these galaxies is simply determined by the amount of time gas has been replenishing the inner regions and triggering star formation. Finally, from this figure it is difficult to determine the dominant formation scenario since, even if mergers have played a (significant) role during the formation history of spiral galaxies, secular evolution could potentially dilute their possible signature in the form of KDCs.

\section{Conclusions}

In these proceedings we have determined the stellar populations (i.e. age) of a sample of kinematically decoupled components found in the SAURON sample of Sa galaxies. We find that they tend to be well aligned and in co-rotation to the galaxies major axis. The younger ones being dynamically colder and more akin to disks. Furthermore, we show that these components can be long-lived. The evidence presented here leads to a picture in which secular evolution seems to play an important role in the history of these galaxies. It also suggests that in galaxies with complex star formation histories, such as spirals, the age and size of the kinematically decoupled component is mainly driven by inflow of gas towards the inner regions, and not necessarily the number of major interaction events the galaxy might have suffered througout its lifetime.

\section{Acknowledgements}

The authors would like to congratulate the organizing committee for a very stimulationg and enjoyable meeting.

\section{References}

Carollo, C. M., Danziger, I. J., Rich, R. M., Chen, X., 1997, ApJ, 491, 545

de Zeeuw, P.T., et al., 2002, MNRAS, 329, 513

Emsellem, E., et al.,, 2001, A\&A, 368, 52

Falcón-Barroso, J., et al., 2006, MNRAS, 369, 529

Forbes, D. A., Franx, M., Illingworth, G. D., 1994, ApJ, 428, L49

Jogee, S., et al., 2004, ApJ, 615, L105

Lacey, C., Cole, S., 1993, MNRAS, 262, 627

Márquez, I., et al., 2003, A\&A, 409, 459

McDermid, R.M., et al., 2006, MNRAS, 373, 906 
Peletier, R.F., et al., 2007, MNRAS submitted

Pfenniger, D., Norman, C., 1990, ApJ, 363, 391

\section{Discussion}

WILD: Do you have any information on the AGN properties of the galaxies?. Do you have enough lines to study narrow line AGNs?.

FALCón-BARroso: We have very little evidence for broad emission lines. Besides that we have very limited information (i.e. emission lines) available to do this type of analysis properly. It would be indeed very interesting to follow this up with many more emission lines.

Chilingarian: Have you checked whether Fe5015 really does trace Fe?. If you look in the NIST database of atomic lines, you will see that this region is populated by Ti lines, and not by iron.

FALCón-BARroso: Fe5015 index is not just a tracer for Fe but, like many other indices, is also coposed by other elements (including $\mathrm{Ti}$ lines). However it is also known that this index is a good tracer of metallicity. At this stage, with our spectral resolution, we cannot distinguish between them.

GALAZ: By measuring $\sigma_{z}$ (disk) in face-on galaxies you can measure directly the massluminosity ratios $(\mathrm{M} / \mathrm{L})$. Are you expecting to do that with some face-on spirals or have you already did it?. At least for your brighter sample...

FALCón-BARRoso: No we do not have enough S/N with SAURON to achieve that measure for our sample. 